\title{
Original
}

\section{Índice de masa corporal pregestacional y ganancia de peso materno y su relación con el peso del recién nacido}

\author{
(Pre gestation body mass index and maternal weight gain, \\ its relationship with new born weight)
}

Manrique Leal-Mateos, Loretta Giacomin, Luis Diego Pacheco-Vargas

\section{Resumen}

Servicio de Obstetricia, Hospital Dr. Rafael Angel Calderón Guardia

Abreviaturas: AEG, Adecuado para Edad Gestacional; CCSS, Caja Costarricense de Seguro Social; GEG, Grande para Edad Gestacional; Hospital Calderón Guardia, HCG; IMC, Índice de masa corporal; PEG, Pequeño para Edad Gestacional.

Correspondencia: Manrique Leal Mateos; email: manrique. leal@gmail.com
Objetivo: Explorar en el sistema de salud nacional la relación existente entre el índice de masa corporal pregestacional y la ganancia de peso materno durante el embarazo, y su relación con el peso del recién nacido. Asimismo, pretende investigar su relación con factores adversos tanto maternos como fetales, al momento de la labor de parto.

Materiales y métodos: Se realizó un estudio observacional analítico de corte transversal para cumplir con los objetivos del estudio. Mediante un muestreo consecutivo se analizaron 360 historias clínicas de pacientes gestantes atendidas en el Servicio de Obstetricia del Hospital "Dr. Rafael Angel Calderón Guardia" entre el 1 de enero y el 28 de febrero de 2008. Las variables maternas relacionadas con el control prenatal que se incluyeron fueron: edad en años cumplidos, peso en kilogramos, talla en metros, índice de masa corporal $\left(\mathrm{kg} / \mathrm{m}^{2}\right)$, ganancia de peso en kilogramos por trimestre y en total durante la gestación. Las variables relacionadas al parto que se analizaron fueron: edad gestacional en semanas cumplidas, tipo de parto, duración de la labor de parto y expulsivo, realización de episiotomía y presencia de desgarros. Las variables neonatales que se estudiaron fueron: sexo; peso al nacer en kilogramos; talla en centímetros; circunferencia cefálica en centímetros; puntuación Apgar a los 5 minutos; clasificación según peso/edad gestacional; presencia de distocia de hombros; lesiones fetales como fracturas, dislocaciones, o parálisis nerviosas, entre otras; necesidad de reanimación neonatal y requerimiento de hospitalización del recién nacido.

Resultados: Si se toma como referencia al grupo con un IMC normal, se puede observar que el porcentaje de recién nacidos pequeños para edad gestacional (PEG) y el de recién nacidos grandes para edad gestacional (GEG), fue significativamente mayor en el grupo con un IMC bajo y con obesidad, respectivamente. Hubo una relación significativa entre la duración de la labor de parto y el IMC pregestacional de embarazada. Asimismo, la presencia de distocia y lesión fetal se observó más frecuentemente en pacientes con obesidad. Se pudo observar también como la ganancia de peso durante la gestación se relaciona también con la antropometría neonatal. No solo se determinó un aumento en el peso, talla y circunferencia cefálica de aquellos productos de madres que ganaron más peso de lo aconsejado por la norma, sino que el porcentaje de productos grande para edad gestacional fue mayor en este grupo. No obstante, al analizar la influencia de la ganancia de peso sobre la labor de parto, no se evidenció una diferencia significativa entre ellas.

Si se toma como referencia al grupo con una ganancia de peso adecuada durante el embarazo, se puede observar que el porcentaje de recién nacidos pequeños para edad gestacional (PEG) fue semejante entre este y los que presentaron una ganancia de peso menor a la recomendada, 
ISSN 0001-6002/2008/50/3/160-167 Acta Médica Costarricense, (O2008 Colegio de Médicos y Cirujanos mientras que el de recién nacidos grandes para edad gestacional (GEG) fue significativamente mayor en el grupo con una ganancia de peso mayor a la aconsejada.

Conclusiones: El presente estudio demostró que el índice de masa corporal y la ganancia de peso durante el embarazo influye sobre el peso de los recién nacidos.

Descriptores: Índice de masa corporal, ganancia de peso materno durante el embarazo, peso del recién nacido, Hospital Rafael Ángel Calderón Guardia.

\section{Abstract}

Aim: To explore the relationship between pregestational body mass index (BMI) and maternal weight gain during pregnancy with the newborns weight in the national health system.

Materials and methods: This is a cross-sectional observational study. Three hundred and sixty medical records from pregnant patients seen at the Obstetrics Service of the Calderon Guardia Hospital were analyzed. Maternal variables related to the prenatal birth control were: age, weight, height, body mass index $\left(\mathrm{kg} / \mathrm{m}^{2}\right)$, weight gain per trimester and total weight gain through pregnancy. Labor and delivery variables included were: gestational age, mode of delivery, labor and expulsive period duration in minutes, episiotomy and presence of perineal tears. Neonatal outcomes analyzed were: sex, birth weight and height, cephalic circumference, 5 minutes Apgar score, wight/age relationship, shoulder dystocia, fetal lesion, need for neonatal resuscitation or newborn hospitalization.

Results: Taking as a reference the group with normal BMI, the percentage of both either small for gestional age (SGA) and large for gestional age (LGA) newborns was higher in the group of low BMI and obesity, respectively. A significant relationship was observed between the length of labor and pregestational BMI. The presence of dystocia and fetal lesion were more frequent in obese patients. Weight gain during gestation was found to be related with neonatal authropometric measurements. The products of mothers whose weight vas higher than advisible weighted more, were taller and had a larger cephalic circumference and in general they were larger than expected for their gestational age.

There was no significant difference between the influence of weight gain during pregnancy and the labor variables. Taking as a reference the group with adequate weight gain during pregnancy, the percentage of SGA and LGA newborns was similar between this group and the one with lower weight gain. In contrast, the incidence of LGA newborn was higher in those patients with greater weight gain.

Conclusion: The present study demonstrated that BMI and weight gain during pregnancy influences the newborn weight.

Key words: Body mass index, weight gain during pregnancy, newborn weight. Rafael Ángel Calderón Guardia Hospital.

Recibido: 8 de abril de 2007

Aceptado: 6 de mayo de 2008
El peso al nacer ha sido considerado como un factor determinante para el futuro de los recién nacidos. Se ha visto como la morbilidad y mortalidad se incrementan según disminuye o aumenta el peso neonatal con base en los valores aceptados como normales. ${ }^{1-4}$

Muchos son los factores que influyen en el crecimiento y desarrollo fetal. Dentro de ellos, existen algunos que pueden ser sujetos a control y modificación por parte del personal de Salud. El estado nutricional pregestacional y ganancia de peso durante el embarazo, por ejemplo, resultan ser algunos de los destacados. ${ }^{3,4}$
Han sido varios los estudios recientes que han confirmado una relación entre la ganancia de peso materno y el peso del recién nacido. No obstante, muchos de ellos han sido realizados en países desarrollados en donde el aspecto cultural, social y económico difiere bastante del nuestro. ${ }^{5-8}$ Por otro lado, existen también otras investigaciones efectuadas en países latinoamericanos que abordan el tema, sin embargo, sus resultados no pueden ser extrapolados a nuestra población, ya que sus sistemas de salud y perfiles epidemiológicos son también distintos a nuestra realidad.9-11 
En virtud de lo anterior, este estudio tiene como objetivo principal explorar dentro del Sistema de Salud Nacional la relación existente entre el índice de masa corporal pregestacional y la ganancia de peso materno durante el embarazo y su relación con el peso del recién nacido. Asimismo, pretende investigar su relación con factores adversos tanto maternos como fetales, al momento de la labor de parto.

\section{Materiales y métodos}

Se realizó un estudio observacional analítico de corte transversal para cumplir con los objetivos del estudio. La presente investigación contó con la aprobación del Comité de Ética del Hospital.

Mediante un muestreo consecutivo se analizaron 360 historias clínicas de pacientes gestantes atendidas en el servicio de Obstetricia del Hospital Dr. Rafael Angel Calderón Guardia (HCG), entre el 1 de enero y el 28 de febrero de 2008.

Se utilizaron criterios de inclusión y exclusión para eliminar la posible influencia de factores de confusión.

Se incluyó en el estudio únicamente a las pacientes que cumplieran con los siguientes criterios: Primigesta; embarazo mayor de 37 semanas; embarazo único; primer control prenatal antes de las 12 semanas de gestación; más de 5 citas de control prenatal.

Además, se excluyó del análisis a toda paciente que presentara: embarazo complicado por enfermedades médicas crónicas o inducidas por este; antecedente de uso de tabaco, alcohol u otra sustancia ilícita que pudiera afectar de forma directa o indirecta el peso fetal final; productos con algún tipo de malformación diagnosticada antes de la labor del parto o durante el nacimiento; ausencia o registro incompleto de alguna variable de interés.

El tamaño de la muestra se calculó con base en los 5755 partos atendidos en el HCG durante 2006 y de acuerdo con una prevalencia máxima de pacientes con un índice de masa corporal inadecuado del $50 \%$. Se estimó un error $\alpha=0,05$ y un error $\beta=0,80$, para lo cual se utilizó el Sistema Statcalc del programa EpiInfo 2002.

La recolección de los datos se realizó mediante una hoja electrónica diseñada para este estudio.

Las variables maternas relacionadas con el control prenatal que se incluyeron fueron: edad en años cumplidos, peso en kilogramos, talla en metros, índice de masa corporal $\left(\mathrm{kg} / \mathrm{m}^{2}\right)$, ganancia de peso en kilogramos por trimestre y en total durante la gestación.

\begin{tabular}{|ccc|}
\hline \multicolumn{2}{|c|}{$\begin{array}{c}\text { Cuadro 1. Ganancia de peso recomendada } \\
\text { durante el embarazo según el IMC } \\
\text { pregestacional, HCG, 2008 }\end{array}$} \\
\hline $\begin{array}{c}\text { Índice de masa corporal } \\
\text { pregestacional }\end{array}$ & Clasificación & $\begin{array}{c}\text { Ganancia } \\
\text { de peso } \\
\text { recomendada } \\
\text { en Kg. }\end{array}$ \\
\hline Menos de 19,8 & Bajo & De 12,5 a 18 \\
De 19,8 a 26,0 & Normal & De 11,5 a 16 \\
De 26,1 a 29,0 & Sobrepeso & De 7 a 11,5 \\
Mayor de 29,0 & Obesidad & Menos de 7 \\
\hline
\end{tabular}

Las variables relacionadas al parto que se analizaron fueron: edad gestacional en semanas cumplidas, tipo de parto, duración de la labor de parto y expulsivo, realización de episiotomía y presencia de desgarros.

Las variables neonatales que se estudiaron fueron: sexo; peso al nacer en kilogramos; talla en centímetros; circunferencia cefálica en centímetros; puntuación apgar a los 5 minutos; clasificación según peso/edad gestacional; presencia de distocia de hombros; lesiones fetales como fracturas, dislocaciones, o parálisis nerviosas, entre otras; necesidad de reanimación neonatal y requerimiento de hospitalización del recién nacido.

Para la categorización de las pacientes según el índice de masa corporal (IMC) pregestacional y la ganancia de peso materno durante el embarazo, se utilizaron los parámetros establecidos por la Academia de Ciencias de los Estados Unidos en 1990, aceptados por la Organización Mundial de la Salud y adaptados por Stotland y colaboradores (Cuadro 1).6, 12,13

Se utilizó el programa EpiInfo 2002 para el procesamiento de los datos. Las variables cualitativas se analizaron mediante frecuencias y proporciones. Los resultados derivados de las variables cuantitativas se expresaron mediante medidas de tendencia central y de dispersión. La relación entre variables cualitativas se efectuó mediante la prueba estadística de Chi cuadrada $\left(\mathrm{X}^{2}\right)$. La diferencia de promedios entre dos grupos se comparó mediante la prueba de t Student. Se utilizaron las pruebas exactas de Fisher y la prueba de "U" de Mann-Whitney, cuando fue requerido. Además, se utilizó el análisis de la varianza (ANOVA) para comparar las diferencias entre los promedios de tres o más grupos. Se utilizó la prueba de Kruskal-Wallis cuando fue necesaria. El nivel de significancia se fijó en $p \leq 0.05$. Se utilizó el programa Excel 2007 para la elaboración de lo cuadros. 


\section{Resultados}

La edad promedio de las muestra fue de 21,5 años (DS+ $4,7)$. La talla y peso promedio de las embarazadas fue de 1,56 centímetros $\left(\mathrm{DS}_{ \pm}+4,7\right)$ y $56,8 \mathrm{Kg} .\left(\mathrm{DS}_{ \pm}+10,8\right)$, respectivamente. El Índice de Masa Corporal (IMC) promedio fue de $23,1 \mathrm{Kg} / \mathrm{m}^{2}(\mathrm{DS} \pm 3,8)$. El 16,7\% $(\mathrm{n}=60)$ presentaron un IMC bajo, el $64,4 \%(n=232)$ un IMC normal, el 7,8 $(n=28)$ sobrepeso y el $11,1 \%(n=40)$ obesidad.

En el Cuadro 2 se detallan las características generales para la población de estudio según la clasificación por IMC. La ganancia de peso trimestral y total de las pacientes durante su embarazo según la clasificación por IMC se muestra en el Cuadro 3.

La edad gestacional promedio de toda la muestra fue de 39,2 semanas $(\mathrm{DS} \pm 1,1)$. El 90,6\% $(\mathrm{n}=326$; IC 95\% 87,1$93,4)$ de la pacientes tuvieron parto vaginal y el $9,4 \%(n=34$; IC 95\% 6,7-13,1) parto por cesárea. El promedio de duración de la labor de parto y el periodo expulsivo para toda la muestra fue de 356,4 minutos (DS $\pm 161,1)$ y 16,2 minutos $(\mathrm{DS} \pm 15,7)$, respectivamente. Al 59,7\% $(\mathrm{n}=215$; IC 95\% 54,4-64,8) de la muestra fue necesario realzar episiotomía, mientras que el 31,4\% ( $n=113$; IC 95\% 26,7-36,5) presentó desgarros perineales. Estas mismas variables, según la clasificación por IMC, se muestran en el Cuadro 4.

El 50,6\% (n=182; IC 95\% 45,3-55,8) de los recién nacidos fueron hombres. El peso promedio en general para todos los recién nacidos fue de 3.216,9 g (DS $\pm 419,2)$. La circunferencia cefálica y talla promedio de los neonatos fue de 34,1 centímetros (DS $\pm 1,5)$ y 49,7 centímetros (DS \pm 1,9), respectivamente. El 99,1\% (n=357; IC 95\% 97,4-99,8) de los recién nacidos presentó un apgar mayor o igual a 7 a los 5 minutos.

Únicamente el 1,1\% (n=4; IC 95\% 0,4-3) y el $0,8 \%$ $(\mathrm{n}=3$; IC 95\% 0,2-2,6) de los neonatos presentaron distocia de hombros y lesiones fetales como consecuencia del parto, respectivamente. Al 22,8\% $(n=82$; IC 95\% 18,6-27,5) fue necesario realizar reanimación neonatal y solo al $4,4 \%$ ( $n=16$; IC 95\% 2,6-7,3) fue preciso hospitalizar. Las variables neonatales según la clasificación por IMC se muestran en el Cuadro 5.

El 87,8\% (n=317; IC 95\% 83,9-91,0) de los recién nacidos fueron clasificados como adecuados para edad gestacional. Únicamente el 3,3\% $(\mathrm{n}=12$; IC 95\% 1,8-5,9) y el $8,9 \%(n=31$; IC 95\% 6,2-12,4) fueron clasificados como pequeños y grandes para edad gestacional, respectivamente. Estas mismas variables, según la clasificación por IMC, se muestran en el Cuadro 6.

Si se toma como referencia al grupo con un IMC normal, se puede observar que el porcentaje de recién nacidos pequeños para edad gestacional (PEG) y el de recién nacidos grandes para edad gestacional (GEG) fue significativamente mayor en el grupo con un IMC bajo y con obesidad, respectivamente (Cuadros 7 y 8). El porcentaje de recién nacidos grandes para edad gestacional (GEG) en el grupo con sobrepeso no fue significativamente mayor.

Únicamente el 41,7\% (n=150; IC 95\% 36,6-47,0) de la muestra tuvo una ganancia de peso adecuada durante su embarazo. El 35,1\% ( $n=126$; IC 95\% 30,1-40,2) tuvo una ganancia de peso menor y el 23,2\% (n=84; IC 95\% 19,1$28,1)$ una ganancia mayor a la recomendada para su IMC pregestacional. La clasificación de ganancia de peso durante la gestación según el IMC se muestra en el Cuadro 9.

\begin{tabular}{|c|c|c|c|c|c|}
\hline \multicolumn{6}{|c|}{ Cuadro 2. Variables maternas según clasificación de IMC, HCG, 2008} \\
\hline & Bajo & Normal & Sobrepeso & Obesidad & Valor de $p$ \\
\hline Edad (años) & $19,7 \pm 4,6$ & $21,4 \pm 4,4$ & $23,1 \pm 4,9$ & $24,4 \pm 5,6$ & $<0,001$ \\
\hline Peso pregestacional (Kg.) & $45,1 \pm 4,9$ & $55,4 \pm 6,1$ & $65,8 \pm 5,8$ & $80,9 \pm 8,8$ & $<0,001$ \\
\hline Talla (metros) & $1,56 \pm 0,07$ & $1,56 \pm 0,06$ & $1,55 \pm 0,06$ & $1,58 \pm 0,05$ & 0,22 \\
\hline IMC pregestacional (Kg./m2) & $18,4 \pm 1,1$ & $22,4 \pm 1,6$ & $27,3+0,76$ & $32,3 \pm 2,3$ & $<0,001$ \\
\hline
\end{tabular}

Cuadro 3. Ganancia de peso materno trimestral y total según clasificación de IMC HCG, 2008

\begin{tabular}{|lccccc|}
\hline & Bajo & Normal & Sobrepeso & Obesidad & Valor de $p$ \\
\hline I trimestre (Kg.) & $2,4 \pm 0,7$ & $2,4 \pm 0,9$ & $2,5 \pm 1,1$ & $2,3 \pm 0,9$ & 0,87 \\
II trimestre (Kg.) & $4,3 \pm 1,4$ & $4,4 \pm 1,5$ & $4,6 \pm 1,8$ & $4,2 \pm 1,6$ & 0,66 \\
III trimestre (Kg.) & $5,3 \pm 1,7$ & $5,5 \pm 1,9$ & $5,7 \pm 2,3$ & $5,4 \pm 2,1$ & 0,91 \\
Total (Kg.) & $12,0+3,8$ & $12,3+4,2$ & $12,8+4,8$ & $11,9+4,3$ & 0,83 \\
\hline
\end{tabular}




\begin{tabular}{|lccccc|}
\hline \multicolumn{7}{|c}{ Cuadro 4. Variables de la labor de parto según clasificación de IMC, HCG, 2008} \\
\hline \\
\hline \multicolumn{7}{|c}{ Bajo } & Normal & Sobrepeso & Obesidad & Valor de $p$ \\
\hline Edad gestacional (sem.) & $39,1 \pm 1,1$ & $39,2 \pm 1,1$ & $39,5 \pm 1,1$ & $39,1 \pm 1,3$ & 0,24 \\
Parto vaginal (\%) & 93,3 & 90,5 & 87,5 & 89,3 & 0,79 \\
Cesárea (\%) & 6,7 & 9,5 & 12,5 & 10,7 & 0,79 \\
Episiotomía (\%) & 68,3 & 56,9 & 70,0 & 50,0 & 0,14 \\
Desgarros (\%) & 31,7 & 30,2 & 30,0 & 42,9 & 0,60 \\
Labor de parto (mín.) & $297,5 \pm 133,3$ & $358,3 \pm 157$ & $389,7 \pm 195$ & $423,6 \pm 163,1$ & 0,002 \\
Expulsivo (mín.) & $15,7 \pm 14,8$ & $15,2 \pm 14,9$ & $20,5 \pm 20,2$ & $20,6 \pm 20,2$ & 0,33 \\
\hline
\end{tabular}

\begin{tabular}{|lccccc|}
\hline \multicolumn{7}{|c|}{ Cuadro 5. Variables del recién nacido según clasificación de IMC, HCG, 2008 } \\
\hline & Bajo & Normal & Sobrepeso & Obesidad & Valor de $p$ \\
\hline Sexo masculino (\%) & 38,3 & 51,7 & 53,6 & 60,0 & 0,16 \\
Peso (gr.) & $3017,6+482,3$ & $3235,9+400,6$ & $3255+300,8$ & $3431,8+427,6$ & $<0,001$ \\
Talla (cm.) & $48,7+2,5$ & $49,7+1,7$ & $49,9+1,8$ & $50,6+1,8$ & $<0,001$ \\
CC (cm.) & $33,7+1,3$ & $34,1+1,6$ & $34,2+0,9$ & $34,3+1,5$ & 0,12 \\
Apgar > 7 a los 5 min. (\%) & 100 & 98,7 & 100 & 100 & 0,70 \\
Distocia & 1,7 & 0,9 & 0,0 & 3,6 & 0,51 \\
Lesión fetal & 1,7 & 0,0 & 0,0 & 7,1 & 0,001 \\
Reanimación & 18,3 & 23,7 & 27,5 & 17,9 & 0,64 \\
Hospitalización & 5,0 & 4,3 & 5,0 & 3,6 & 0,98 \\
\hline
\end{tabular}

Dada la influencia del IMC sobre el peso neonatal demostrada en los cuadros de párrafos anteriores, se seleccionó únicamente a aquellas pacientes con un IMC pregestacional normal $(\mathrm{n}=232,64 \%)$ para explorar la relación entre la ganancia de peso durante la gestación y el peso del recién nacido. El objetivo de esto fue eliminar la posible influencia de IMC sobre esta relación.

La ganancia de peso materno durante el embarazo de forma trimestral y total se muestra en el Cuadro 10.

Las variables neonatales y de la labor de parto, según la ganancia de peso durante el embarazo se muestran en los Cuadros 11 y 12. En el 12 se puede observar la existencia de una diferencia estadísticamente significativa entre los pesos neonatales y la ganancia de peso durante la gestación.

Si se toma como referencia al grupo de madres con una ganancia de peso adecuada durante el embarazo, se puede observar que el porcentaje de recién nacidos PEG fue semejante entre este $(2,1 \%, \mathrm{n}=2)$ y las que presentaron una ganancia de peso menor a la recomendada $(4,7 \%, n=4)$, $\mathrm{p}=0,40$.
Nuevamente, si se toma como referencia al grupo de madres con una ganancia de peso adecuada durante el embarazo, se puede observar que el porcentaje de recién nacidos GEG fue mayor en las que presentaron una ganancia de peso mayor a la recomendada $(28,6 \%, \mathrm{n}=10)$ que en el de referencia $(7,2 \%, n=7), p<0,001$.

\section{Discusión}

Antes de comentar los resultados obtenidos es importante mencionar algunos factores que pueden influir en la interpretación de los resultados.

En primer lugar, el estudio no mide causalidad, únicamente relación o asociación entre las variables independientes y dependientes seleccionadas.

Por otro lado, al ser un estudio de base hospitalaria y no poblacional, los resultados son aplicables únicamente a las pacientes atendidas en el HCG. 
Por último, al utilizar historias clínicas de los expedientes médicos y tarjetas de control prenatal de las pacientes como fuente de datos, el estudio no se encuentra libre de sesgos de información.

Con base en este concepto, y después de controlar los posibles factores de confusión mediante la restricción de ciertas variables, el estudio demostró que el índice de masa corporal influye sobre el peso de los recién nacidos. Aunque la diferencia es discreta entre los grupos con un IMC normal y con sobrepeso, esta es más marcada y significativa entre las pacientes con un IMC normal y aquellas con un IMC bajo o con obesidad. Asimismo, se demostró que el porcentaje de recién nacidos pequeños y grandes para edad gestacional es mayor en los grupos con un IMC bajo y con obesidad, respectivamente.

Con respecto a la labor de parto, consideramos que las diferencias encontradas en la duración de esta pueden deberse a la constitución fetal. En pacientes con obesidad, en quienes la duración de la labor de parto fue significativamente más prolongada, se pudo observar que la antropometría de los recién nacidos fue también mayor. Caso contrario ocurrió con aquellas con un IMC bajo, en quienes la labor de parto no solo duró menos, sino que los

\section{Cuadro 6. Clasificación de peso para edad gestacional según de IMC, HCG, 2008}

\begin{tabular}{|lcccc|}
\hline & Bajo & Normal & Sobrepeso & Obesidad \\
\hline $\begin{array}{l}\text { Pequeño para edad } \\
\text { gestacional (\%) }\end{array}$ & 10,0 & 2,6 & 0,0 & 0,0 \\
$\begin{array}{l}\text { Adecuado para edad } \\
\text { gestacional (\%) }\end{array}$ & 83,3 & 88,8 & 95,0 & 78,6 \\
$\begin{array}{l}\text { Grande para edad } \\
\text { gestacional (\%) }\end{array}$ & 6,7 & 8,6 & 5,0 & 21,4 \\
\hline
\end{tabular}

Cuadro 7. Clasificación pequeño para edad gestacional según la presencia de un IMC bajo materno, HCG, 2008

\begin{tabular}{|lccccc|}
\hline & \multicolumn{2}{c}{ Bajo } & Normal & $\begin{array}{c}\text { Valor } \\
\text { de } p\end{array}$ \\
\hline $\begin{array}{l}\text { Clasificación peso / } \\
\text { edad gestacional }\end{array}$ & $\mathrm{n}$ & $\%$ & $\mathrm{n}$ & $\%$ & \\
$\begin{array}{l}\text { Pequeño para edad } \\
\text { gestacional (\%) }\end{array}$ & 6 & 10,7 & 6 & 2,8 & - \\
$\begin{array}{l}\text { Adecuado para edad } \\
\text { gestacional (\%) }\end{array}$ & 50 & 89,3 & 206 & 97,2 & - \\
\hline Total & 56 & 100 & 212 & 100 & 0,01 \\
\hline
\end{tabular}

$\mathrm{OR}=4,1(\mathrm{IC} 95 \% 1,1-15,2)$ productos fueron de menor tamaño y peso. Los fetos grandes para edad gestacional quizás necesiten mayor tiempo y contracciones más "fuertes" para progresar durante su labor de parto. Otra hipótesis propuesta para este fenómeno es la acumulación de tejido blando en la pelvis de las mujeres obesas, que podría de cierta forma "estrechar" el diámetro del canal de parto y sumarse al tamaño fetal para prolongar la labor. ${ }^{14}$

También, la presencia de distocia y lesión fetal se observó más frecuentemente en pacientes con obesidad. Aunque en el primer tipo de complicación la diferencia entre los grupos no fue estadísticamente significativa, esto puede deberse a un problema de potencia estadística más que clínico, ya que los resultados son congruentes a los observados en estudios semejantes.

Por otro lado, se pudo observar también como la ganancia de peso durante la gestación se relaciona con la antropometría neonatal. No solo se observó un aumento en el peso, talla y circunferencia cefálica de aquellos productos de madres que ganaron más peso de lo aconsejado por la norma, sino que el porcentaje grandes para edad gestacional fue mayor en este grupo.

\begin{tabular}{|c|c|c|c|c|c|}
\hline \multicolumn{6}{|c|}{$\begin{array}{l}\text { Cuadro 8. Clasificación grande para edad } \\
\text { gestacional según la presencia de obesidad } \\
\text { materna, HCG, } 2008\end{array}$} \\
\hline & \multicolumn{2}{|c|}{ Obesidad } & \multicolumn{2}{|c|}{ Normal } & $\begin{array}{l}\text { Valor } \\
\text { de } p\end{array}$ \\
\hline $\begin{array}{l}\text { Clasificación peso / } \\
\text { edad gestacional }\end{array}$ & $n$ & $\%$ & $n$ & $\%$ & \\
\hline $\begin{array}{l}\text { Grande para edad } \\
\text { gestacional }(\%)\end{array}$ & 6 & 21,4 & 20 & 8,8 & - \\
\hline $\begin{array}{l}\text { Adecuado para edad } \\
\text { gestacional (\%) }\end{array}$ & 22 & 78,6 & 206 & 91,2 & - \\
\hline Total & 28 & 100 & 226 & 100 & 0,03 \\
\hline
\end{tabular}

$\mathrm{OR}=2,8(\mathrm{IC} 95 \% 0,9-8,4)$

Cuadro 9. Clasificación de ganancia de peso durante el embarazo según IMC, HCG, 2008

\begin{tabular}{|lcccc|}
\hline & Menor & Adecuada & Mayor & Valor de $\mathrm{p}$ \\
\hline Bajo & 48,3 & 45,0 & 6,7 & \\
Normal & 39,2 & 45,7 & 15,1 & \\
Sobrepeso & 12,5 & 32,5 & 55,0 & \\
Obesidad & 3,6 & 14,3 & 82,1 & $\mathrm{p}<0,001$ \\
\hline
\end{tabular}


No obstante, al analizar la influencia de la ganancia de peso sobre la labor de parto, no se evidenció una diferencia significativa entre ellas. Lo anterior plantea la discusión sobre la relevancia clínica de esta variable al momento de la labor. Si bien la ganancia excesiva de peso durante la gestación se asocia a una mayor morbilidad materna durante el embarazo, resulta cuestionable, a la luz de los resultados obtenidos, su importancia clínica al momento propio del parto.

Sin embargo, es prematuro descartar totalmente la posibilidad de una relación. Para esto, se necesitan estudios epidemiológicos con métodos diferentes al utilizado para confirmar los resultados.

Por último, conviene resaltar que únicamente el 64,4\% de las pacientes presentaron un IMC pregestacional adecuado, e incluso, solamente el $41 \%$ de ellas ganaron durante su embarazo un peso adecuado para su respectivo IMC. El médico de cabecera, sea general o gineco-obstetra, debe preparar a la mujer para su posible gestación y orientarla durante su embarazo para que prevenga o corrija conductas o hábitos de riesgo que puedan alterar la evolución normal de este y llevarlo a complicaciones posteriores.
En este caso específico, el médico debe aconsejar a la mujer para que evite índices de masa corporal que se alejen del recomendado y prevenga ganancias de peso menores o excesivas durante su gestación, que puedan significar un riesgo para ella y su futuro hijo.

La futura madre debe conocer su peso actual y llegar al embarazo con un peso adecuado, según su contextura. La malnutrición no corregida previa al embarazo se asocia a poca ganancia de peso y con esto a un aumento en la morbilidad neonatal. Durante la adolescencia este problema adquiere especial importancia, ya que los requerimientos son mayores a los de la mujer adulta.

\begin{tabular}{|lcccc|}
\hline \multicolumn{5}{|c|}{$\begin{array}{c}\text { Cuadro 10. Ganancia de peso materno } \\
\text { trimestral y total, HCG, 2008 }\end{array}$} \\
\hline & Menor & Adecuada & Mayor & $\begin{array}{c}\text { Valor } \\
\text { de } p\end{array}$ \\
\hline I trimestre (Kg.) & $1,6 \pm 0,5$ & $2,6 \pm 0,4$ & $3,7 \pm 1,2$ & $<0,001$ \\
II trimestre (Kg.) & $2,9 \pm 0,8$ & $5,0 \pm 0,6$ & $6,6 \pm 0,9$ & $<0,001$ \\
III trimestre (Kg.) & $3,7 \pm 1,1$ & $6,2 \pm 0,8$ & $8,3 \pm 1,3$ & $<0,001$ \\
Total (Kg.) & $8,2+2,3$ & $13,9+1,4$ & $18,8+2,3$ & $<0,001$ \\
\hline
\end{tabular}

\section{Cuadro 11. Variables de la labor de parto según la ganancia de peso durante el embarazo, HCG, 2008}

\begin{tabular}{|lcccc|}
\hline & Menor & Adecuada & Mayor & Valor de $p$ \\
\hline Parto vaginal (\%) & 95,6 & 86,6 & 88,6 & 0,09 \\
Cesárea (\%) & 4,4 & 13,2 & 11,4 & 0,09 \\
Episiotomía (\%) & 57,1 & 55,7 & 60,0 & 0,90 \\
Desgarros (\%) & 34,1 & 23,6 & 40,0 & 0,11 \\
Labor de parto (mín.) & $359,5 \pm 150,8$ & $348,4 \pm 159,3$ & $384,3 \pm 167,9$ & 0,52 \\
Expulsivo (mín.) & $16,4 \pm 15,9$ & $13,9 \pm 12,5$ & $15,6 \pm 12,1$ & 0,54 \\
\hline
\end{tabular}

Cuadro 12. Variables del recién nacido según ganancia de peso durante la gestación, HCG, 2008

\begin{tabular}{|lcccc|}
\hline & Menor & Adecuado & Mayor & Valor de $p$ \\
\hline Peso (gr.) & $3153,7 \pm 361,7$ & $3210,6 \pm 400,1$ & $3526,1 \pm 377,5$ & $<0,001$ \\
Talla (cm.) & $49,4+1,6$ & $49,8+1,7$ & $50,6+1,7$ & 0,004 \\
CC (cm.) & $33,7+1,2$ & $34,1+1,8$ & $34,8+1,2$ & 0,002 \\
Apgar $>$ 7 a los 5 min. (\%) & 98,1 & 97,1 & 100,0 & 0,34 \\
Distocia & 0,0 & 1,9 & 0,0 & 0,30 \\
Lesión fetal & 0,0 & 0,0 & 0,0 & - \\
Reanimación & 23,1 & 25,5 & 20,0 & 0,79 \\
Hospitalización & 4,4 & 3,8 & 5,7 & 0,88 \\
\hline
\end{tabular}


Por otro lado, y si bien no fue el objetivo del estudio, es importante resaltar que la obesidad se asocia a complicaciones médicas como la hipertensión o la diabetes, que aumentan de forma importante el riesgo perinatal. Además, algunos estudios indican que el ambiente intrauterino influye sobre riesgo futuro de obesidad en los hijos de madres con sobrepeso. ${ }^{15}$ De hecho, los hijos de madres obesas clasificados como grandes para edad gestacional tienen 3 veces más probabilidad de presentar obesidad a la edad de 7 años. ${ }^{16}$

Por lo tanto, se insiste en que la labor del médico antes y durante un embarazo debe ser dinámica y continua. Resulta fundamental comunicar y educar a la mujer en edad fértil sobre los factores que aumentan el riesgo materno y perinatal y que pueden ser reducidos o controlados en esta etapa de su vida.

\section{Referencias}

1. McIntire DD, Bloom SL, Casey BM, Leveno KJ. Birth weight in relation to morbidity and mortality among newborn infants. N Engl J Med 1999; 340:1234-1238.

2. Alexander GR, Kogan M, Bader D, Wally C., Marilee A., Joanne M. US birth weight/gestational age-specific neonatal mortality: 19951997 rates for whites, hispanics and blacks. Pediatrics 2003; 111: e61.

3. American College of Obstetricians and Gynecologists: Intrauterine growth restriction. Practice Bulletin No. 12, January 2000b.

4. American College of Obstetricians and Gynecologists: Fetal macrosomia. Practice Bulletin No. 22, November 2000a.

5. Usha TS, Hemmadi S, Bethel J, Evans J. Outcome of pregnancy in a woman with an increased body mass index. BJOG 2005; 112: 768772 .

\section{Simposio Internacional de Cáncer de Tiroides}

\section{8 y 29 de Agosto de 2008}

20 Horas de Carrera Profesional

$$
\text { Recertificación Médica }
$$

$$
\begin{gathered}
\text { Inscripción e información: } \\
\text { Médicos Nacionales } \$ 100 \\
\text { Enfermeras Profesionales } \$ 50 \\
\text { Estudiantes } \$ 50
\end{gathered}
$$

\section{Colegio de Médicos y Cirujanos}

Recepción 2232-3433 ext 114 y 115

\section{Banco de Costa Rica}

$\phi$ colones cuenta $\mathrm{n}^{\circ}$ 001-01-917-0006569-2

$\$$ dólares cuenta $n^{\circ}$ 001-02-917-0006287-1 Enviar por fax copia del vaucher nombre completo:
6. Stotland NE, Cheng YW, Hopkins LM, B. Caughey. Gestational weight gain and adverse neonatal outcome among term infants. Obstet Gynecol 2006; 108:635-643.

7. DeVader SR, Neeley HL, Myles TD, Leet TL. Evaluation of gestational weight gain guidelines for women with normal prepregnancy body mass index. Obstet Gynecol 2007; 110: 745-751.

8. Cedergren MI. Optimal gestational weight gain for body mass index categories. Obstet Gynecol 2007; 110: 759-764.

9. Martínez MT, Matienzo G, Williams S, Cruz PR; Gómez AM. Ganancia de peso materno: relación con el peso del recién nacido. Rev Cubana Obstet Ginecol 1999; 25:114-117.

10. Grados FM, Cabrera R, Díaz J. Estado nutricional pregestacional y ganancia de peso materno durante la gestación y su relación con el peso del recién nacido. Rev Med Hered 2003; 14:128-133.

11. Lagos R, Espinoza R, Orellana JJ. Estado nutritivo materno inicial y peso promedio de sus recién nacidos a término. Rev Chil Nutr 2004; 31: 52-57.

12. Institute of Medicine (U.S.), Subcommittee on nutritional status and weight gain during pregnancy and subcommitteeon dietary Intake and nutrient supplements during pregnancy. Nutrition during pregnancy. Washington (DC): National Academies Press; 1990. Pp 25-36.

13. WHO. Maternal anthropometry for prediction of pregnancy outcomes: Memorandum from a USAID/ WHO/PAHO/ Mother Care meeting. Bull WHO 1991; 69: 523-532.

14. Vahratian A, Zhang J, Troendle JF, Savitz DA, Siega-Riz AM. Maternal prepregnancy overweight and obesity and the pattern of labor progression in term nulliparous women. Obstet Gynecol 2004; 104: 943-951.

15. Reece AE. Perspectives on obesity, pregnancy and birth outcomes in the United States: The scope of the problem. Am J Obstet Gynecol 2008; 23-27.

16. Salsberry PJ, Reagan PB. Dynamics of early childhood overweight. Pediatrics 2005; 116:1329-38.

\section{Seminario de Diabetes Mellitus}

13 y 14 de noviembre de 2008

16 Horas de Carrera Profesional

10 Créditos Recertificación Médica

$$
\begin{gathered}
\text { Inscripción e información } \\
\text { Profesionales } \phi 40000 \\
\text { Estudiantes } \phi 30000
\end{gathered}
$$

Colegio de Médicos y Cirujanos

Recepción 2232-3433 ext 114 y 115

Cuentas colones $n^{\circ} 119497-6$

Cuenta dólares $\mathrm{n}^{\circ}$ 119498-4

\section{Fundación para el desarrollo del Hospital Nacional de Niños.}

Enviar por fax copia del vaucher nombre completo: 